\title{
Erratum to: Dual-phase amyloid PET: hitting two birds with one stone
}

\author{
Valentina Garibotto $^{1} \cdot$ Silvia Morbelli ${ }^{2}$ - Marco Pagani ${ }^{3,4}$
}

Published online: 24 May 2016

(C) Springer-Verlag Berlin Heidelberg 2016

Erratum to: European Journal of Nuclear Medicine and Molecular Imaging

DOI 10.1007/s00259-016-3393-6

The original version on this paper contained an error.

The names of all authors were displayed incorrectly. The names and surnames of the authors are inversed. Correct presentation is given below.

Valentina Garibotto

Silvia Morbelli

Marco Pagani

The online version of the original article can be found at http://dx.doi.org/ 10.1007/s00259-016-3393-6.

Marco Pagani

marco.pagani@istc.cnr.it

1 Division of Nuclear Medicine and Molecular imaging, Department of Medical Imaging, Geneva University and Geneva University Hospitals, Geneva, Switzerland

2 Nuclear Medicine Unit, IRCCS San Martino - IST, Department of Health Sciences, University of Genoa, Genoa, Italy

3 Institute of Cognitive Sciences and Technologies, CNR, Rome, Italy

4 Department of Nuclear Medicine, Karolinska Hospital, Stockholm, Sweden 